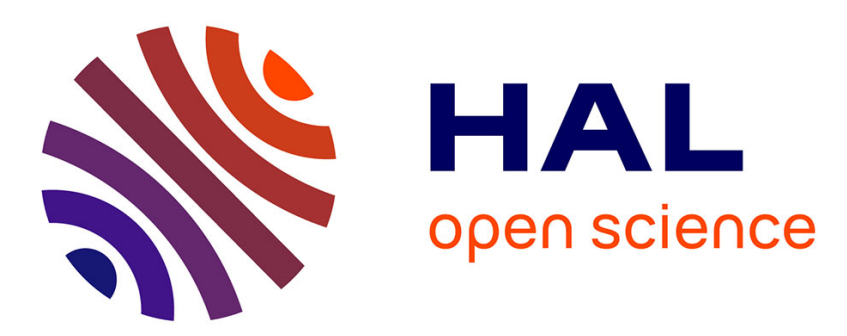

\title{
La visibilité du genre dans des revues de sociologie du travail
}

\author{
Nicky Le Feuvre, Pierre Bataille, Laura Morend
}

\section{To cite this version:}

Nicky Le Feuvre, Pierre Bataille, Laura Morend. La visibilité du genre dans des revues de sociologie du travail: Comparaisons France et Grande-Bretagne (1987-2012). Cahiers du Genre, 2013, Rétrospectives, 54, pp.121-150. 10.3917/cdge.054.0121 . hal-01861246

\section{HAL Id: hal-01861246 \\ https://hal.science/hal-01861246}

Submitted on 24 Aug 2018

HAL is a multi-disciplinary open access archive for the deposit and dissemination of scientific research documents, whether they are published or not. The documents may come from teaching and research institutions in France or abroad, or from public or private research centers.
L'archive ouverte pluridisciplinaire HAL, est destinée au dépôt et à la diffusion de documents scientifiques de niveau recherche, publiés ou non, émanant des établissements d'enseignement et de recherche français ou étrangers, des laboratoires publics ou privés. 


\section{La visibilité du genre dans des revues de sociologie du travail}

Article in Cahiers du Genre · January 2013

DOI: $10.3917 /$ cdge.054.0121

3 authors, including:

Some of the authors of this publication are also working on these related projects:

Project $\quad$ ANR Woman (Woman in Management) View project

Project Gendered globalization of the legal professions View project 


\title{
La visibilité du genre dans des revues de sociologie du travail
}

\author{
Comparaisons France et Grande-Bretagne \\ (1987-2012)
}

\section{Nicky Le Feuvre, Pierre Bataille et Laura Morend}

\section{Résumé}

Le travail a constitué un objet privilégié des premières recherches académiques sur les rapports sociaux de sexe en France et les 'études genre' y sont restées marquées par cet héritage. Peut-on affirmer pour autant que les dimensions sexuées du travail sont désormais intégrées au cœur de la sociologie du travail et que les spécialistes du genre y sont reconnu·e·s à part entière ? Pour répondre à ces questions, nous proposons une analyse comparative de l'évolution du nombre d'articles consacrés au 'genre du travail' dans deux revues de sociologie du travail en France et en GrandeBretagne. Nous montrons que la part de recherches portant sur les dimensions sexuées du travail à trouver place dans ces revues est beaucoup plus réduite en France qu'outre-Manche, et ceci tout au long de la période étudiée. La 'cécité au genre' qui perdure au sein de la sociologie du travail en France n'occulte pas seulement la réalité laborieuse des femmes de ce pays, elle invisibilise et marginalise également les (femmes) spécialistes du genre au sein de ce champ académique. ${ }^{1}$

\footnotetext{
${ }^{1}$ L'analyse des résultats présentés ici s'est effectuée en partie au sein de l’IP6 du Pôle de recherche national (PRN) LIVES «Surmonter la vulnérabilité. Perspective du parcours de vie », financé par le Fonds national suisse de la recherche scientifique (FNS). Nous remercions le Comité de lecture et les expert·e $\cdot \mathrm{s}$ anonymes des Cahiers $d u$ genre pour leurs commentaires éclairés sur une version précédente de ce texte.
} 
SOCIOLOGIE DU TRAVAIL — ÉTUDES GENRE — REVUES — ÉDITION — FRANCE — GRANDE-BRETAGNE

Dans ce numéro consacré aux évolutions des usages du concept de genre au fil des ans, le présent article vise à éclairer les conditions de reconnaissance et de généralisation des approches analytiques inspirées par une prise en compte des dimensions sexuées du monde du travail. Plus de cinquante ans après les premières recherches menées explicitement sur les expériences féminines du travail, rémunéré et domestique (par exemple Myrdal, Klein 1956 ; Guilbert 1966), il s'agit d'évaluer à quel point les problématiques de recherche développées dans le sillage de la deuxième vague du Mouvement de libération des femmes se sont imposées au cœur ou sont restées en marge des préoccupations de la sociologie du travail. Pour ce faire, nous avons retracé les évolutions en matière de prise en compte du genre dans deux contextes nationaux contrastés, notamment en ce qui concerne le processus d'institutionnalisation des études genre dans le monde académique (Griffin 2005a) ; la France et la GrandeBretagne.

Notre étude se divise en deux parties. Dans un premier temps, nous proposons d'analyser le degré d'ouverture aux 'questions de genre' de deux revues généralistes ${ }^{2}$ traitant des questions de travail : Sociologie du travail (SDT), pour la France et Work, Employment \& Society (WES), pour la Grande-Bretagne. Il s'agira alors de mesurer l'attention et la place accordées (ou pas) à la dimension sexuée du travail et de l'emploi dans les articles publiés dans ces revues et d'évaluer l'évolution quantitative de

\footnotetext{
${ }^{2}$ Dans cet article, nous désignons les revues Sociologie du travail (SDT) et Work, Employment \& Society (WES) comme des revues 'généralistes', alors que les revues dont l'intitulé comporte une référence directe au genre - Travail, genre et sociétés (TGS), Cahiers du genre (CDG), Gender, Work \& Organization (GWO) sont nommées 'spécialistes'. En effet, bien que s’intéressant prioritairement au travail, $S D T$ et WES revendiquent explicitement le statut de revues généralistes, le travail y étant conçu comme une entrée thématique privilégiée pour l'analyse des principes généraux de fonctionnement des sociétés avancées. Sur le fond, il pourrait évidemment en aller de même pour les revues qui portent explicitement sur le genre...
} 
celle-ci au fil du temps. L'adoption d'une perspective comparative a l'avantage de révéler les éventuelles spécificités sociétales dans ce que Margaret Maruani (2001) a appelé « la longue marche à petits pas » vers une reconnaissance du genre au sein de la sociologie du travail française. Cette marche a-t-elle été aussi longue et fastidieuse dans le contexte britannique ? A-t-elle suivi des tracés similaires ou emprunté des chemins diversifiés dans chacun de ces contextes ? Comment cette marche s'est-elle articulée à la création de revues spécialisées 'genre et travail'?

Outre une restitution essentiellement descriptive de la visibilité des dimensions sexuées du travail dans ces revues scientifiques ${ }^{3}$, nous nous intéresserons, dans un deuxième temps, aux pratiques des chercheur $\cdot \mathrm{e} \cdot \mathrm{s}$ en matière de valorisation des recherches sur les thématiques 'genre et travail'. Les femmes sontelles objectivement surreprésentées parmi les auteur·e·s d'articles scientifiques portant sur les dimensions sexuées du travail ? Le sont-elles au même point dans les deux pays, dans les deux types de revues et au même degré au fil du temps ? Les articles sur le genre du travail donnent-ils à voir des pratiques plus collectives et collaboratives d'écriture que ceux publiés sur le travail en général ? Ces pratiques sont-elles stables ou fluctuantes ? Ici, en complément des données concernant les revues généralistes, nous analyserons également les pratiques observées au sein de deux revues spécialisées (Travail, genre et sociétés (TGS), pour la France ; Gender, Work \& Organization (GWO), côté britannique) ${ }^{4}$.

\footnotetext{
${ }^{3}$ Faute de pouvoir faire justice aux données à notre disposition dans un seul article, nous réservons à une publication ultérieure l'analyse de l'évolution des objets, des thèmes et des problématiques analytiques qui ont retenu l'attention des chercheur·e·s s'intéressant aux dimensions sexuées du travail au fil du temps.

${ }^{4}$ En réalité, bien qu'étant matériellement gérées et éditées dans des contextes nationaux spécifiques, toutes les revues analysées ici attirent des contributions qui dépassent très largement les seul $\cdot \mathrm{e} \cdot \mathrm{s}$ chercheur $\cdot \mathrm{e} \cdot \mathrm{s}$ travaillant dans leurs pays d'implantation. Si nous les désignons en fonction de leurs pays d'édition, il convient de garder à l'esprit leur inscription dans des 'aires culturelles et linguistiques' plus larges.
} 


\section{Objectifs et méthodologie}

Pour mener à bien ce projet, nous avons mobilisé des données plutôt faciles d'accès, mais fastidieuses à traiter et donc peu exploitées de manière systématique à ce jour, à savoir le contenu des revues scientifiques en sociologie du travail. Notre démarche s'inscrit dans la lignée des tentatives antérieures de quantification de la prise en compte du genre dans les recherches menées sur le travail, en France (Maruani 2001 ; Lallement 2003). Elle vient également compléter les recherches historiographiques visant à retracer l'apparition des premiers articles scientifiques sur les femmes et le genre dans une série de revues en sciences sociales (CLIO 2002).

Nous avons donc saisi dans une base de données les références de l'ensemble des articles publiés, hors recensions et comptes rendus de lecture, depuis 1987 (date de création de WES) pour les revues généralistes et depuis 1994 (date de création de GWO) pour les revues spécialisées en études genre. Nous exploitons ici un total de 2559 références. Pour chacun de ces articles, nous avons effectué plusieurs classifications, concernant à la fois les caractéristiques de leurs auteur·e·s (sexe, publiant seul·e ou en collaboration) et le contenu de l'article (titre, mots clés, date de parution). Précisons d'emblée que le recensement des 'articles genre' présenté ici tient compte de la totalité des publications portant sur les dimensions sexuées du travail, sans opérer de distinction a priori en fonction des choix lexicaux ou des perspectives théoriques adoptés. Ainsi, outre les articles qui comportent une mention spécifique du mot 'genre / gender' dans l'intitulé ou les mots clés, nous avons également comptabilisé comme 'articles genre' des textes qui n'utilisent pas ce terme, mais qui portent explicitement sur les questions de division sexuelle du travail, de ségrégation sexuée des emplois, de féminisation des métiers, d'articulation des temps de vie, d’inégalités sexuées de rémunération, de politiques d'égalité, de mobilisation collectives sexuées (grèves de femmes, par exemple) et de travail domestique ou de care. Bien que la désignation 'article genre' soit quelque peu anachronique dans le cas français, puisque le terme 'genre' ne s'est pleinement imposé dans la production scientifique francophone qu’au milieu des années 1990 (Fougeyrollas- 
Schwebel et al. 2003 ; Bereni 2011), nous l'avons adopté par simple facilité rédactionnelle. Rappelons qu'il s'agit ici d'opérer un recensement quantitatif de la visibilité des recherches portant sur les dimensions sexuées du travail, non de réaliser une analyse de l'évolution des problématiques mobilisées pour en rendre compte.

Tableau 1. Nombre d'articles analysés dans chacune des revues recensées

\begin{tabular}{|l|c|c|c|c|}
\hline & $\begin{array}{c}\text { Sociologie } \\
\text { du travail } \\
(S D T)\end{array}$ & $\begin{array}{c}\text { Work, } \\
\text { Employment } \\
\text { \& Society } \\
(\text { WES })\end{array}$ & $\begin{array}{c}\text { Travail, } \\
\text { genre et } \\
\text { sociétés } \\
(T G S)^{5}\end{array}$ & $\begin{array}{c}\text { Gender, Work } \\
\text { \& } \\
\text { Organization } \\
(\text { GWO })\end{array}$ \\
\hline $\begin{array}{l}\text { Période } \\
\text { couverte }\end{array}$ & $1987-2012$ & $1987-2012$ & $1995-2012$ & $1994-2012$ \\
\hline $\begin{array}{l}\text { Nombre } \\
\text { d'articles } \\
\text { recensés }\end{array}$ & 665 & 968 & 456 & 470 \\
\hline Total & \multicolumn{2}{|c|}{1633} & \multicolumn{2}{|c|}{926} \\
\hline
\end{tabular}

\section{Brève présentation des revues sélectionnées}

Notre échantillon est loin d'épuiser la totalité de la production scientifique en sociologie du travail dans les deux pays retenus pour l'analyse. De plus, dans la mesure où elles sont issues de cultures scientifiques spécifiques (Galtung 1982), il paraît difficile d'affirmer que ces revues sont strictement équivalentes. Néanmoins, autant pour les revues généralistes que pour les publications spécialisées, nous avons essayé de circonscrire des espaces semblables de diffusion des connaissances scientifiques sur le travail dans les contextes français (francophone) et britannique (anglophone). Malgré la trentaine d'années qui séparent leurs débuts, il nous semble que les revues Sociologie du travail (créée en 1959) et Work, Employment \& Society (créée en 1987) occupent aujourd'hui des espaces intellectuels équivalents au sein de leurs aires linguistiques respectives. On est immédiatement frappé par la proximité des objectifs revendiqués par ces deux

\footnotetext{
${ }^{5}$ Afin de bénéficier d’une échelle temporelle comparable dans les deux pays, nous avons recensé les articles parus, pour la période 1995-1997, dans les Cahiers du MAGE, publication qui a précédé la création de la revue Travail, genre et sociétés.
} 
revues - l'appréhension des enjeux majeurs de société par le biais de l'analyse du travail —, par leurs inscriptions disciplinaires - ancrage en sociologie, mais ouverture et dialogue avec des disciplines proches - , et par leur volonté proclamée de dialogue avec les acteurs et actrices du monde extra-académique.

Du côté des deux revues spécialisées, la périodicité de création est beaucoup plus proche et témoigne d'un calendrier relativement homogène d'institutionnalisation des études genre dans les universités européennes. Le premier numéro de la revue Gender, Work \& Organization paraît en 1994, précisément au moment de la création du Groupement de recherche (GDR) Marché du travail et genre (MAGE) au sein du CNRS, en France. Les travaux de ce réseau national sont d'abord diffusés au moyen d'une publication interne: Les Cahiers du MAGE (19951997), avant la création d'une revue commerciale, intitulée Travail, genre et sociétés, éditée d'abord chez L'Harmattan (19992004), puis chez Armand Colin (2005-2008) et, depuis 2009, à La Découverte. Les finalités et raisons d'être de GWO et TGS sont également proches : Gender, Work \& Organization se définit comme " la première revue à rassembler un éventail large de recherches inter- et multidisciplinaires dans ce domaine dans un nouvel espace international de débat et d'analyse. Des contributions sont invitées de toutes les perspectives disciplinaires, dont l'anthropologie, l'histoire, les sciences économiques, le droit, la philosophie, la science politique, la psychologie et la sociologie " ${ }^{6}$. De son côté, Travail, genre et sociétés se présente comme : "une revue pluridisciplinaire, internationale et ouverte aux différents courants qui traversent ces domaines de recherche " ${ }^{7}$. Toutefois, comme nous le verrons plus loin, malgré la similitude de leurs objectifs et calendriers de création, chacune de ces revues s’inscrit dans un contexte académique spécifique, caractérisé par une différence très significative dans la prise en compte des dimensions sexuées du travail dans les revues généralistes établies antérieurement en sociologie du travail.

\footnotetext{
${ }^{6}$ Site Internet de GWO, consulté le 7 janvier 2013.

${ }^{7}$ Site Internet de TGS, consulté le 7 janvier 2013.
} 


\section{Questions de recherche}

Le présent article vise d'abord à éclairer les modalités de l'institutionnalisation des études genre dans différents contextes sociétaux ; il s'efforce ensuite de saisir une part de l'expérience des chercheur.e.s investi·e.s dans ce champ, notamment en matière de reconnaissance professionnelle.

Sur le premier point, notre analyse comparée des revues nous amène à nuancer le récit qui tend à s'imposer un peu partout en Europe aujourd'hui, et qui présente le mainstreaming du genre comme le résultat d'un cheminement homogène et linéaire, faisant passer progressivement l'ensemble des pays européens de la dénégation / stigmatisation à la reconnaissance / intégration des approches analytiques élaborées par des générations successives de chercheur·e·s féministes. Ce récit s'inspire parfois des recherches sociologiques menées sur le processus d'institutionnalisation des études genre, mais en trahit souvent l'esprit. En effet, contrairement à ce qui en est parfois compris, ces recherches ne visent pas à caractériser un processus qui se présenterait partout de la même manière (avec simplement plus ou moins de 'retard' et des 'résistances' plus ou moins ardues à surmonter), mais cherche bien à rendre compte des spécificités sociétales relativement durables en la matière.

Ainsi la sociologue finlandaise Harriet Silius (2002) a-t-elle élaboré une typologie du processus d'institutionnalisation académique des études genre, comportant cinq modalités distinctes : le modèle 'activiste' se caractérise par le maintien durable de liens étroits entre le mouvement de libération des femmes et les activités pédagogiques et de recherche; des enseignements informels (principalement de conscientisation) se mettent en place à l'intérieur ou à l'extérieur des universités et quelques recherches ('action') se développent de manière éparse, sans financement ou reconnaissance académiques. Un modèle, dit 'd'intégration', est susceptible de se substituer au précédent ; il se caractérise par la mise en œuvre d'un nombre limité d'enseignements sur le genre dans le cadre universitaire, principalement sous la forme de cours optionnels au sein des disciplines traditionnelles et par le financement ponctuel de quelques programmes 'fléchés' de recherche. Il se distingue d'un troisième 
modèle d'institutionnalisation, dit 'd'affirmation', marqué par la multiplication des voies de diffusion des connaissances sur le genre, soit au sein des disciplines traditionnelles, soit par le biais de structures spécifiques (départements, centres de recherche, filières de formation, diplômes, revues académiques spécialisées), soit par ces deux canaux à la fois. Un quatrième modèle, dit de 'professionnalisation', se caractérise par la consolidation des possibilités d'enseignement, de recherche (y compris en doctorat) et de publication sur le genre, phénomène accompagné de la création en nombre significatif de postes statutaires fléchés, réservés aux spécialistes des études genre. La cinquième et dernière modalité de cette typologie est celle de 'l'autonomisation' ou de la 'disciplinarisation' : le champ des études genre reçoit les mêmes attributions de moyens et la même reconnaissance institutionnelle que les autres disciplines universitaires et perd progressivement son statut d'exception.

La tentation est forte de voir chacune de ces modalités d'institutionnalisation comme la suite (logique) de la modalité précédente, dans une perspective quelque peu évolutionniste. Toutefois, il paraît important d'insister sur le fait que ces différentes modalités d'institutionnalisation ne se succèdent pas nécessairement de manière linéaire dans tous les pays et que les 'retours en arrière' sont toujours possibles. Cette typologie permet ainsi de nuancer l'idée d'une progression linéaire de reconnaissance de l'importance du genre dans l'analyse sociologique du travail, puisqu'elle pointe l'existence d'une certaine "dépendance au sentier » (path dependancy) dans le processus d'institutionnalisation des études genre ${ }^{8}$. En appliquant ce mode de raisonnement au contenu des revues de sociologie du travail, nous voudrions illustrer la nature foncièrement contingente et non téléologique de l'institutionnalisation des études genre dans les mondes académiques, ici britannique et français.

Ensuite, il s'agit de montrer que chacune des modalités spécifiques d'institutionnalisation des études genre façonne de

\footnotetext{
${ }^{8}$ Initialement développée pour comprendre les processus d'innovation technique (David 1985), puis mobilisée pour analyser la diffusion des politiques publiques (Pierson 1994), l'approche en termes de «dépendance au sentier » part de l'hypothèse que les conditions sociohistoriques d'apparition d'une innovation influencent durablement les modalités de sa diffusion et appropriation.
} 
manière précise ce que cela signifie (en termes de statut, de conditions d'emploi, de reconnaissance, de légitimité, etc.) d'être 'chercheur·e spécialiste du genre' dans tel ou tel contexte sociétal. Ainsi, alors que le fait de travailler sur le genre peut constituer un handicap insurmontable à la carrière académique là où les études genre sont peu ou faiblement institutionnalisées, la consolidation de ce champ est tout à fait susceptible d'ouvrir de nouvelles perspectives de carrière aux chercheures féministes (Griffin 2005b ; Bereni 2012), voire de contribuer à la féminisation plus généralisée du corps professoral. Nous avançons ici l'hypothèse selon laquelle la présence / absence des recherches sur le genre au sein des revues académiques généralistes les plus prestigieuses constitue un indicateur assez précieux des chances de carrière qui s'offrent aux spécialistes du genre dans différents contextes sociétaux. Cela permet d'analyser les conditions de production des 'articles genre’ de manière renouvelée.

\section{Vous avez dit gender?}

Le premier résultat qui émerge de l'analyse comparative des revues généralistes est particulièrement frappant : au cours de la période 1987-2012, la revue britannique Work, Employment \& Society a publié presque cinq fois plus d'articles sur les dimensions sexuées du travail que son homologue française, Sociologie du travail (Tableau 2). Dans de telles circonstances, il n'est guère étonnant que les recherches en matière de genre du travail aient progressivement acquis la réputation d'être d'inspiration anglo-saxonne en France !

Tableau 2. Répartition thématique des articles publiés dans WES et $S D T, 1987-2012$

\begin{tabular}{|c|c|c|c|c|c|}
\hline & \multicolumn{2}{|c|}{ Nombre } & \multicolumn{2}{|c|}{$\%$} & \multirow[b]{2}{*}{ Total } \\
\hline & $\begin{array}{c}\text { Hors } \\
\text { Genre }\end{array}$ & Genre & $\begin{array}{l}\text { Hors } \\
\text { Genre }\end{array}$ & Genre & \\
\hline Sociologie du travail & 623 & 42 & 93,7 & 6,3 & 665 \\
\hline $\begin{array}{l}\text { Work, Employment } \\
\text { \& Society }\end{array}$ & 770 & 198 & 79,5 & 20,5 & 968 \\
\hline
\end{tabular}

Note de lecture : Sur la période 1987-2012, 6,3\% des articles publiés dans la revue Sociologie du Travail font référence aux dimensions sexuées du travail. 
D’emblée, ces résultats confirment les observations effectuées par Margaret Maruani pour la période antérieure à celle analysée ici :

[...] de 1959 (date de la création de la revue Sociologie du travail) à 1999 (date de la parution du numéro spécial intitulé "Tables 1959-1999»), on observe que les entrées 'Femmes', 'Genre', 'Rapports sociaux de sexe' ou 'Division sexuelle du travail' n'apparaissent nulle part dans les indexes thématiques. Et pour cause : sur les 862 articles publiés en l'espace de quarante ans, 18 traitent de ces questions - dont quatre regroupés dans le seul numéro spécial consacré au sujet «Travail des femmes et famille » (n³/1984). Ainsi, dans une publication considérée comme la revue légitime, sorte de temple de la sociologie du travail, les femmes qui représentent $46 \%$ du monde du travail constituent $2 \%$ de la production scientifique (Maruani 2001, p. 44-45).

En bref, cette première analyse permet d'observer que, depuis la fin des années 1980, il est rare de trouver un numéro de Work, Employment \& Society qui ne comporte aucun 'article genre', alors que cela constitue la norme dans Sociologie du travail. Ces résultats soulèvent de nombreuses interrogations quant à la situation manifestement plus favorable à la prise en compte des dimensions sexuées du travail au sein de la sociologie britannique, en comparaison du cas français. Si nous ne manquons pas d'hypothèses pour rendre compte de cette intégration différenciée des approches genre (Andriocci, Le Feuvre 2006), le dispositif méthodologique adopté ici limite nécessairement la capacité de validation empirique de nos intuitions. Indéniablement utile pour révéler la variabilité sociétale du processus de mainstreaming du genre dans le monde académique, notre démarche impose certaines précautions interprétatives. Si nous pouvons affirmer sans réserve que la sociologie du travail britannique s'est révélée nettement plus ouverte et réceptive aux recherches sur les dimensions sexuées du travail que cela n'a été le cas en France, l'identification des mécanismes à l'origine de cette différence s'avère délicate. Cela est d'autant plus le cas que nous manquons de données sur les procédures de soumission, expertise et sélection des articles en vigueur au sein de ces revues à différentes périodes de leur histoire (cf. Lallement 2003). Avec les seules informations à notre disposition, rien ne nous permet de savoir si les spécialistes français·e·s du 
genre ont tout simplement soumis moins de propositions d'articles à Sociologie du travail que leurs homologues britanniques ne l'ont fait à Work, Employment \& Society, donnant lieu au déséquilibre constaté en la matière. Rien ne nous permet non plus de savoir si les éventuels 'articles genre', hypothétiquement soumis en proportion équivalente de part et d'autre de la Manche, auraient connus des sorts différents lors des procédures d'évaluation menées au sein de chacune des revues généralistes analysées ici.

Pour affiner un peu l'analyse de la place accordée aux dimensions sexuées du travail dans les revues généralistes, nous avons opéré un découpage chronologique plus fin au sein de la base de données. Nous disposons de peu de repères chronologiques discriminants pour scinder l'époque de «la sociologie au sein de l'université de masse » (Chenu 2002, p. 51) dans les deux pays. Néanmoins, à partir de la littérature sur l'institutionnalisation des études genre (Griffin 2004, 2005a ; Silius 2005), nous avons retenu l'idée d'un calendrier approximatif du processus d'intégration des perspectives féministes dans les cursus académiques. Nous avons donc distingué trois périodes, correspondant grosso modo aux différentes étapes d'institutionnalisation des études genre identifiées dans le contexte français ${ }^{9}$ (Le Feuvre, Andriocci 2004, 2005). Afin d'obtenir des périodes de durée à peu près équivalente, nous avons retenu les années 1987-1993 comme celles de l'amorce timide et plutôt confidentielle des études genre, marquée par la création des premiers enseignements et par les premiers postes académiques fléchés (en nombre très limité) au sein des disciplines traditionnelles en France. La deuxième période, allant de 1994 à 2002, correspond à celle du développement de ce champ d'études, culminant par un regain d'intérêt médiatique pour les inégalités de sexe qui a précédé l'adoption de la loi sur la Parité. La dernière période, de 2003 à 2012, correspond à une consolidation, voire à une amorce de professionnalisation des études genre (Bereni 2012), dont la création récente de l'Institut du Genre au CNRS pourrait constituer l'un des signes concrets.

\footnotetext{
${ }^{9}$ La chronologie britannique n'est pas forcément identique à celle observée en France, mais cela n'affecte pas directement les analyses proposées ici.
} 
Ce découpage chronologique permet de constater que la prise en compte des dimensions sexuées du travail dans les revues généralistes n’a pas connu une évolution linéaire au cours de la période étudiée ici. En prenant les deux revues ensemble, on constate bien une augmentation progressive du nombre 'd'articles genre' au fil du temps (Tableau 3), mais avec un fléchissement assez net (-6\%) au cours de la période intermédiaire (19942002). En réalité, l'évolution quantitative de ces publications ne s'est pas déroulée de la même manière dans les deux pays ; le fléchissement des années 1994-2002 est principalement le fait de la revue Sociologie du travail, qui connaît un 'creux' impressionnant 'd'articles genre' à cette époque, alors qu'un cinquième des articles publiés dans WES portent sur cette thématique au cours de la même période.

La deuxième période (1994-2002) correspond à la création des deux nouvelles revues spécialisées sur la question genre et travail dans les deux pays, GWO pour la Grande-Bretagne et TGS pour la France. À partir des tendances esquissées dans le Tableau 3, on peut voir que la création de ces revues spécialisées s'inscrit dans deux dynamiques sociétales radicalement différentes. Dans le cas britannique, on peut faire l'hypothèse que c'est bien le dynamisme constaté en matière d'analyse sexuée du travail, y compris dans les numéros successifs de $W E S$, qui est à l'origine du projet de création de nouvelles revues, centrées sur le genre. Dans le court texte de présentation des objectifs et finalités de GWO, qui figure en l'état quasiment identique sur le site web de sa maison d'édition depuis 1994, il est précisé :

La conscience du genre comme dimension centrale de la vie quotidienne et de la société se généralise. Les sciences sociales reflètent, à juste titre, cet intérêt croissant pour le genre et cela est particulièrement le cas dans le domaine du travail et des organisations, précisément là où notre revue se positionne ${ }^{10}$.

\footnotetext{
${ }^{10}$ Site Internet de GWO, consulté le 7 janvier 2013.
} 
Tableau 3. Évolution de la proportion d'articles publiés dans SDT et WES intégrant une perspective de genre, 1987-2012

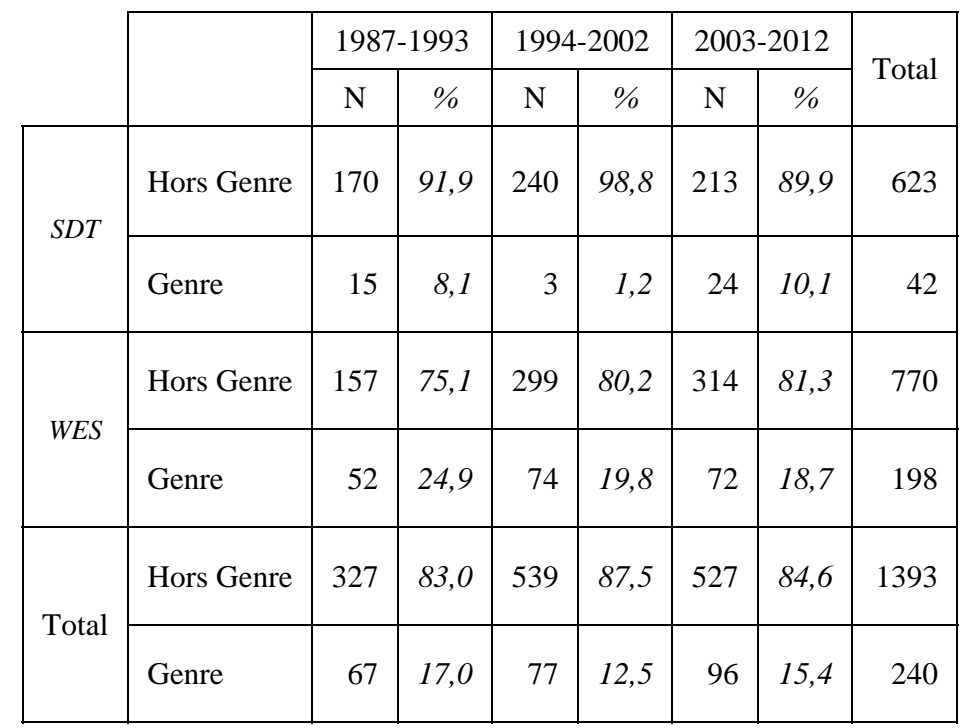

Note de lecture : Sur la période 1994-2002, 3 articles publiés dans la revue Sociologie du travail portent sur la dimension sexuée du travail, représentant $1,2 \%$ du total des articles publiés dans cette revue sur cette période.

Dans le cas français, si l'on se réfère aux objectifs initiaux de $T G S$, c'est moins le désir de capitaliser une évolution favorable observée par ailleurs que la nécessité de compenser l’invisibilité d'une telle dynamique de recherche au cœur de la discipline et singulièrement au fil des pages de $S D T$, qui constitue la principale motivation de création des revues spécialisées. Ainsi :

Poser la question de la différence des sexes dans les sciences sociales du travail et inviter à la réflexion sur le travail dans le champ des recherches sur le genre, décrypter, à partir des hiérarchies, des divisions et des segmentations qui parcourent le monde du travail, le statut des hommes et des femmes dans la société, et poser ainsi la question de la différence des sexes: telle est l'hypothèse fondatrice de Travail, genre et sociétés ${ }^{11}$.

Par ailleurs, la mise en parallèle de l'évolution de l'offre éditoriale et des pratiques observées dans les revues généralistes pour la période 1994-2002 laisse apparaître que la création d’une

\footnotetext{
${ }^{11}$ Site Internet de TGS, consulté le 7 janvier 2013.
} 
revue privilégiant la perspective de genre ne semble pas avoir eu les mêmes effets sur les revues généralistes dans les deux contextes nationaux. Ainsi, du moins dans un premier temps, la création de revues centrées sur les analyses en termes de genre et où les questions de travail occupent une place importante ${ }^{12}$, semble avoir amplifié la 'cécité au genre' (gender blindness) déjà manifeste dans le contexte français, notamment au sein de $S D T$. En effet, on constate une baisse de près de 7 points du taux 'd'articles genre' dans cette revue entre la première période et les années 1994-2002. Bien qu'il soit impossible de conclure à une 'migration' en masse des spécialistes français·es du genre de l'espace académique généraliste aux nouvelles revues spécialisées, il est intéressant de noter que, de l'autre côté de la Manche, le lancement de GWO ne semble avoir eu qu'une influence marginale sur la présence de publications portant sur les dimensions sexuées du travail au sein de WES...

Précisons que ce traitement différencié des questions de genre dans les revues généralistes ne semble nullement être lié au degré de féminisation de leurs équipes de rédaction. Nous constatons sans surprise que les femmes sont depuis toujours très largement majoritaires au sein des instances de décision des revues spécialisées en genre et travail (voir Annexe). Toutefois, elles occupent également depuis longtemps et à parité — ou presque des fonctions rédactionnelles au sein des revues généralistes en sociologie du travail, reflétant le taux relativement élevé de féminisation des postes académiques en Sciences de l'Homme et de la Société dans les deux pays (Commission européenne 2009). A priori, du côté des revues généralistes, il n'existe aucune relation directe entre le nombre de femmes dans les comités de rédaction et la part 'd'articles genre' publiés. On ne peut toutefois pas manquer de souligner la composition très majoritairement masculine du comité d'orientation de Sociologie du travail, et même exclusivement masculine en 2012, et de noter qu'il ne comporte aucun·e spécialiste du genre, contrairement à l'instance

\footnotetext{
${ }^{12}$ Outre TGS, nous pensons ici aux Cahiers du Gedisst, dont le premier numéro paraît en 1991 et qui devient Cahiers du Genre en 1999 et à la revue CLIO : Histoire, femmes, sociétés, publiée aux Presses universitaires du Mirail, à partir de 1995.
} 
équivalente (International Advisory Board) du côté de Work, Employment \& Society.

\section{Les pratiques rédactionnelles autour du genre du travail}

À la suite de ce recensement quantitatif, nous avons souhaité analyser les pratiques rédactionnelles des auteur·e·s des 'articles genre' sous deux angles particuliers. Premièrement, il nous a semblé important de vérifier si les publications sur les dimensions sexuées du travail étaient plus souvent le fait de femmes que d'hommes. Deuxièmement, nous avons souhaité appréhender les éventuelles spécificités dans les conditions de production des articles en question, notamment en matière de signature. Ces deux interrogations reflètent notre intérêt pour les carrières académiques au féminin (Le Feuvre 2010 ; Bataille 2013) et visent à explorer certains des arguments avancés pour expliquer le 'retard de carrière' des femmes universitaires en général (Fassa, Kradolfer 2010) et des femmes spécialistes du genre de surcroît (Ollagnier, Solar 2006). Ces arguments renvoient, tout d'abord, aux choix peu rentables que les femmes chercheures effectueraient en matière de domaines de recherche (le genre étant vu comme l'objet peu rentable par excellence) et, en deuxième lieu, au caractère prétendument plus collectif des productions scientifiques des femmes ou des recherches d'inspiration féministe. Or, dans un monde académique où la logique de l'évaluation individuelle serait en train de prendre le pas sur des pratiques collaboratives antérieures (Puig de la Bellacasa 2002), les femmes ou les spécialistes du genre se trouveraient pénalisé· e·s par une tendance à négliger la valorisation personnelle de leurs travaux dans des revues scientifiques de prestige.

Nous avons voulu traquer les traces de ces pratiques potentiellement spécifiques aux recherches sur le genre du travail, en regardant de plus près les pratiques de signature des 'articles genre', en comparaison avec les publications qui ne s'intéressent pas aux dimensions sexuées du travail. Outre leur appartenance sexuée, les spécialistes du genre ont-elles/ils davantage tendance à publier à plusieurs, plutôt que seul·e·s ? Si oui, cette tendance s'est-elle maintenue au fil du temps et de manière identique dans les deux pays ? Les résultats des différentes 
analyses menées sur ces thèmes sont présentés dans les graphiques ci-dessous.

Tout d'abord, pour les deux revues généralistes et sur l'ensemble de la période couverte ici, nous pouvons confirmer une représentation plus importante des femmes parmi les auteur $\cdot \mathrm{e} \cdot \mathrm{s}$ d'articles genre par rapport à l'ensemble des textes publiés dans SDT et WES (Graphique 1).

Graphique 1. Variations de la part de femmes parmi les auteur.e.s en fonction de la nature des articles publiés dans les revues $S D T$ et WES, 1987-2012

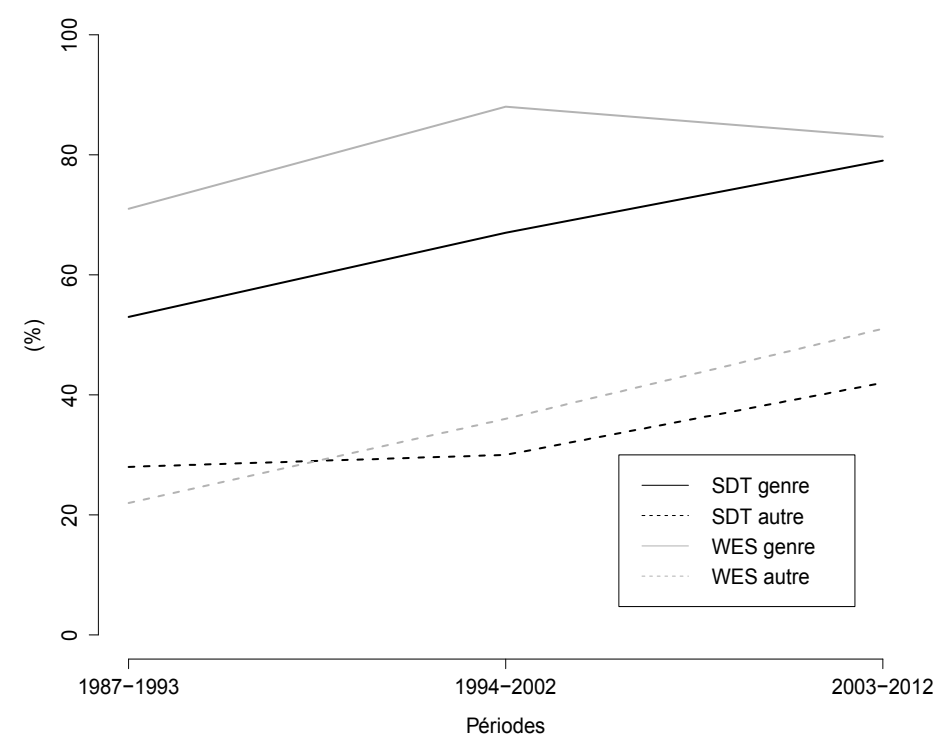

Note de lecture : En 1987-1993, 71 \% des ‘articles genre’ publiés dans la revue WES comptaient au moins une femme auteure.

Les chances de trouver au moins une femme parmi les auteur·e·s sont nettement plus élevées quand il s'agit d'un article portant sur les dimensions sexuées du travail que sur n'importe laquelle des autres thématiques couvertes par ces revues. Cet écart est particulièrement marqué dans le cas de WES, laissant penser que le fait de travailler sur le genre a pu constituer un atout pour l'accès des chercheures à la publication dans cette revue, surtout dans la première phase. De fait, à la fin des 
années 1980, seulement $20 \%$ des 'articles hors genre' parus dans WES comportait au moins une auteure femme, alors que cela était le cas pour plus de 70 \% des 'articles genre'.

Les choses paraissent plus nuancées dans le cas de Sociologie du travail. Tout d'abord, alors que les 'articles genre' parus dans WES sont très clairement l'apanage des femmes au cours de toute la période couverte, cela n'est pas aussi clairement le cas pour ceux publiés dans Sociologie du travail. En effet, en début de période, près de la moitié des (rares) 'articles genre' parus dans cette revue francophone étaient rédigés par des hommes, écrivant seuls ou à plusieurs. C'est seulement à partir du début des années 2000 que la part des 'articles genre' publiés dans Sociologie du travail, signés ou co-signés par au moins une femme, dépasse la barre de $60 \%$. En revanche, que ce soit en France ou au Royaume-Uni, les femmes sont très largement représentées parmi les auteur·e·s des articles parus dans les deux revues spécialisées, où plus de $80 \%$ des articles publiés compte au moins une auteure femme.

En fin de compte, la variabilité de l'attention accordée aux dimensions sexuées du travail dans les revues généralistes s'articule à deux enjeux de visibilité des femmes au travail. D’une part, comme l'a relevé Margaret Maruani (2001), il y a la question de la visibilité (ou de l'invisibilité) des expériences de la moitié de la population active des sociétés européennes actuelles. D'autre part, et cela recoupe davantage nos préoccupations de recherche, il y a la question de la visibilité de la production scientifique des femmes au sein même du monde académique. En d'autres termes, en publiant nettement plus d'articles sur les dimensions sexuées du travail, WES offre une vitrine bien plus fournie que son homologue francophone aux recherches menées par des femmes sur le sujet du travail. Derrière les questions de visibilité ou d'invisibilité sociologique des femmes travailleuses, il existe bel et bien une question de discrimination à l'égard des femmes chercheuses dans ce domaine. De manière paradoxale, les effets discriminatoires de l'occultation des recherches sur les dimensions sexuées du travail dans les pages de $S D T$ se sont intensifiés au fil du temps. En effet, dans le cas français, alors que ces travaux étaient auparavant davantage menés (ou publiés) par des hommes en France 
qu'en Grande-Bretagne, on assiste à une féminisation progressive de la signature des 'articles genre' au fil du temps. Autrement dit, en publiant peu 'd'articles genre' dans la première période, la revue $S D T$ éliminait autant d'auteurs hommes potentiels que d'auteures femmes. Par contre, au fur et à mesure que ce champ scientifique devient clairement l'apanage des femmes, la faible quantité 'd'articles genre' publiés tend à exclure ces dernières des pages de la revue. Quand on connaît l'importance des indicateurs bibliométriques pour le déroulement des carrières académiques, on comprend que le degré d'attention accordée aux dimensions sexuées du travail dans les revues généralistes (et globalement considérées comme plus 'prestigieuses' que les revues spécialisées) peut exercer une influence indirecte sur les carrières académiques au féminin.

\section{De l'individualisation des pratiques de valorisation... dans le contexte français}

Parallèlement à ces premières analyses, nous nous sommes intéressés au caractère plus ou moins collectif de l'entreprise de publication d'articles dans les différentes revues étudiées. En reprenant le découpage chronologique opéré précédemment, on s'aperçoit que les pratiques de rédaction seul·e ou en collaboration varient assez significativement au fil du temps, et pas forcément dans le même sens dans les deux pays (Graphique 2). Ainsi, alors qu'environ $80 \%$ des articles publiés dans SDT (qu'ils portent ou non sur les dimensions sexuées du travail) sont signés par un·e auteur·e unique, la rédaction collective et la cosignature deviennent des pratiques de plus en plus courantes au fil du temps dans WES : pour la dernière période, la moitié des articles publiés dans cette revue sont co-signés. Il semblerait que cela représente bien un phénomène plus large, puisque, bien que de moindre ampleur, les différences constatées en matière de signature individuelle ou collective des articles dans les revues généralistes se retrouvent également dans TGS et GWO (représentées en pointillés sur le Graphique 2).

Pour compléter ces premières remarques, on peut préciser que, dans le cas de WES, il n'y a aucune différence sexuée dans 
les pratiques rédactionnelles - hommes et femmes s'orientent progressivement vers la co-signature de manière identique au fil du temps et ceci qu'ils et elles s'intéressent aux dimensions sexuées du travail ou pas. Par contre, dans le cas de Sociologie du travail, les femmes ont historiquement été nettement plus souvent co-auteures des articles qu'elles publiaient que les hommes (auteurs seuls), avec, toutefois, un alignement de leur part sur les pratiques masculines en fin de période.

Graphique 2. Variations de la part d'articles écrits en solo dans les différentes revues, 1987-2012

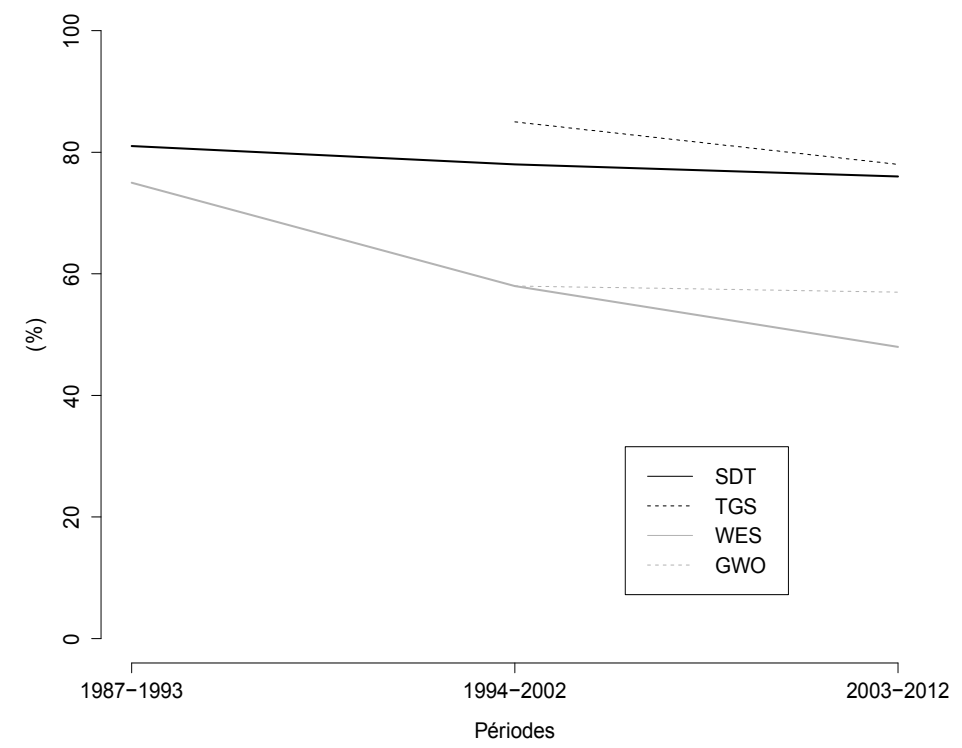

Note de lecture : En 2003-2012, $76 \%$ des articles publiés dans SDT étaient signés par un·e auteur·e seul·e (contre $48 \%$ pour WES).

Comment se situent les 'articles genre' par rapport à ces tendances? Sur l'ensemble de la période couverte ici (19872012), les articles portant sur les dimensions sexuées du travail sont plus souvent signés par deux ou plusieurs auteur·e·s que l'ensemble des textes publiés dans SDT ou dans WES. Toutefois, l'écart est faible (environ $7 \%$ ) et vient nuancer l'idée largement répandue d'une tendance nettement plus marquée au travail collectif dans le champ des études genre (Ollagnier, Solar 2006). 
De plus, selon le contexte sociétal, on observe d'importantes variations dans le temps quant à cette pratique d'écriture collective. Ainsi, alors que les pratiques de publication deviennent globalement plus collectives au fil du temps dans le contexte britannique, on observe une individualisation progressive de la production scientifique sur les dimensions sexuées du travail au sein de Sociologie du travail (Graphique 3). À la fin des années 1980, les (rares) 'articles genre' publiés dans cette revue étaient nettement plus souvent signés par plusieurs auteur·e·s que les textes 'hors genre'. À la fin de la période, cette différence a complètement disparu, au point que les articles sur les dimensions sexuées du travail sont aujourd'hui aussi souvent signés par un·e auteur·e seul·e que l'ensemble des articles publiés dans cette revue. Il paraît d'autant plus difficile d'attribuer cette évolution à la généralisation de principes 'anglo-saxons' d'évaluation 'individualisante' de la productivité scientifique que, comme nous l'avons vu, la co-signature s'impose de plus en plus souvent dans le contexte britannique, autant pour les 'articles genre' qu'en général.

Graphique 3. Variations de la part d'articles genre écrits en solo dans les revues généralistes, 1987-2012

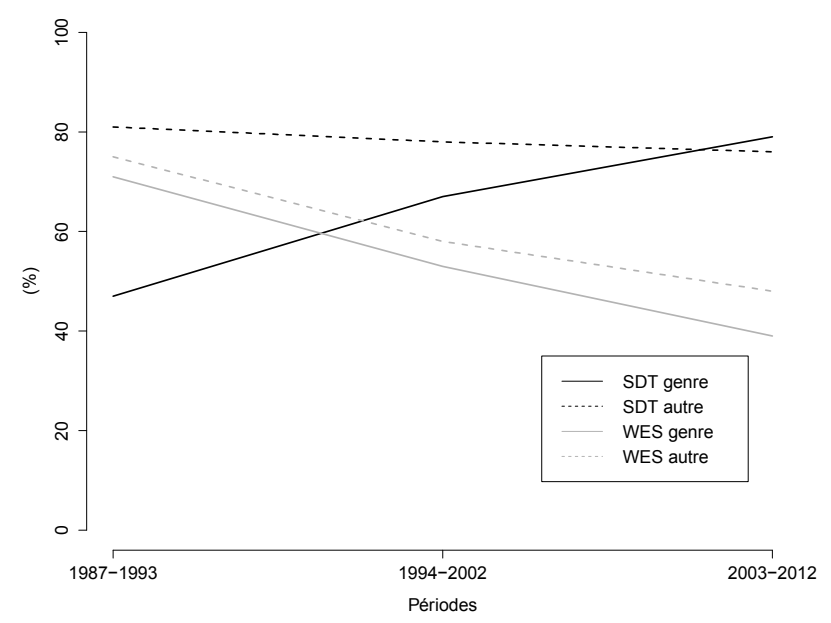

Note de lecture : En 2003-2012, 79 \% des 'articles genre’ publiés dans SDT étaient signés par un·e auteur·e seul·e (contre $39 \%$ pour WES). 


\section{Discussion et conclusions}

À partir des données à notre disposition, il est difficile d'établir les raisons des différences sociétales observées ici, que ce soit en matière de présence / absence des recherches sur les dimensions sexuées du travail dans des revues généralistes en sociologie du travail ou en matière de signature collective / individuelle des 'articles genre'. Tout au plus peut-on esquisser quelques hypothèses, qui demanderaient évidemment vérification au moyen d'autres enquêtes.

Une première série d'hypothèses concerne les conditions sociohistoriques de fondation des revues généralistes (et spécialistes) en sociologie du travail dans les deux pays analysés ici ; une seconde série d'hypothèses concerne le cercle vertueux (ou vicieux) qui découle de la modalité précise d'institutionnalisation des études genre qui caractérise la France et la GrandeBretagne à un moment donné de leurs histoires respectives ; une troisième et dernière série d'hypothèses concerne les procédures d'appel à contribution en vigueur dans les différentes revues, généralistes ou spécialistes, en sociologie du travail dans chaque contexte sociétal.

En avançant de telles hypothèses, nous écartons d'emblée la piste explicative des spécificités socioculturelles en matière de relations entre les sexes (Ozouf 1995 ; Badinter 2003). Au contraire, il nous paraît plus éclairant d'insister sur les diverses formes de "dépendance au sentier» qui marquent le fonctionnement des instances académiques dans différents contextes sociétaux (Musselin 2005) et qui se reflètent nécessairement dans les pratiques éditoriales des revues scientifiques. Ainsi, en premier lieu, il nous paraît probable que le contexte sociohistorique de création des deux revues généralistes en sociologie du travail explique, au moins en partie, les écarts constatés en matière de prise en compte des dimensions sexuées du travail depuis la fin des années 1980.

Alors que la revue Sociologie du travail est fondée bien avant les bouleversements intellectuels et socio-sexués qui marquent l'après mai 1968 et l'émergence du Mouvement de libération des femmes (MLF) en France, la création plus tardive de WES semble avoir constitué, en soi, l'une des réalisations marquantes 
du «bras académique » (Ezekiel 1994) du Mouvement de libération des femmes dans le contexte britannique. Si le cas de WES frappe par l'importance explicitement accordée à l'analyse du travail féminin dès le tout premier numéro paru, il est également intéressant de constater le maintien de cette ligne directrice au fil du temps. En dépit de changements réguliers dans la composition et la localisation institutionnelle des comités de rédaction, environ un cinquième des articles publiés dans WES portent sur les dimensions sexuées du travail et cette proportion varie peu avec les années. A contrario, en l'absence d'un engagement fondateur sur ces thématiques et malgré l'intégration (tardive) d'une (seule) éminente spécialiste du genre dans ses instances rédactionnelles ${ }^{13}$, le travail féminin et les problématiques genre peinent à s'imposer dans les pages de Sociologie du travail, même lors de la phase actuelle d'intégration relative des études genre dans le paysage académique français.

En dépit d'un domaine thématique et d'objectifs tout à fait comparables, les spécificités dans la mise en scène de ces deux revues viennent étayer une telle piste explicative. Côté français, la référence aux 'pères fondateurs' occupe une place tout à fait centrale dans la présentation actuelle de Sociologie du travail, et cela est également le cas dans un ouvrage récent consacré à l'histoire de cette revue (Borzeix, Rot 2010). Ainsi, le site web de Sociologie du travail affirme que cette revue, publiée avec le concours du CNRS, s'inscrit dans une lignée composée exclusivement de sociologues du travail... de sexe masculin :

Depuis sa création en 1959 par Michel Crozier, Jean-Daniel Reynaud, Alain Touraine, Jean-René Tréanton, sous le patronage de Georges Friedmann et de Jean Stoetzel, cette revue trimestrielle de langue française poursuit une double orientation. Revue spécialisée d'un domaine - celui du travail - elle tient aussi à rester une revue de sociologie générale, en prise sur les problèmes d'actualité, ouverte au dialogue avec les disciplines voisines. Elle publie des résultats de recherche empirique, des travaux théoriques, des numéros thématiques pluridisciplinaires,

\footnotetext{
${ }^{13}$ Il n'est sans doute pas inutile de préciser que la seule spécialiste du genre au sein du Comité de rédaction actuelle de SDT (Catherine Marry, DR CNRS au Centre Maurice Halbwachs), a également occupé des fonctions de direction au sein du GDR MAGE, et fait également partie du Comité de rédaction élargi de TGS.
} 
des dossiers-débats et des symposiums susceptibles d'alimenter, scientifiquement, le débat sur des questions de société contemporaine ${ }^{14 .}$

Depuis les travaux fondateurs d'Andrew Abbott (1999, 2001), nous savons que la manière dont les universitaires mobilisent des références à l'histoire des disciplines et aux aïeux prestigieux nous apprend davantage des enjeux actuels du monde académique qu'elle ne nous renseigne sur des filiations véritables en matière d'idées ou d'approches. Il n'empêche, on peut imaginer que cette manière d'inscrire $S D T$ dans un lignage intellectuel où les dimensions sexuées du travail ont occupé une place négligeable (Maruani 2001 ; Lallement 2003 ; Borzeix, Rot 2010) doit nécessairement renvoyer un message aux spécialistes du genre quant à l'intérêt que le comité de rédaction est susceptible de porter à leurs travaux. De ce point de vue, le contraste avec l'auto-présentation de Work, Employment \& Society est frappant.

Effectivement, côté britannique, les individus (non moins prestigieux) impliqués dans la fondation de WES sont totalement effacés du "récit des origines », au bénéfice d'une orientation problématique explicite. Les futures contributions à la revue sont invitées à s'intéresser aux " aspects moins débattus » du travail dont le Comité de rédaction souhaite encourager l'exploration scientifique. Dans l'éditorial du tout premier numéro de cette revue, cette invitation à l'examen de thématiques marginalisées jusqu'alors en sociologie du travail s'accompagne d'un appel au déplacement du regard des chercheur·e·s vers de nouveaux objets d'étude, dont, en premier lieu, le travail féminin. D’emblée, le premier rédacteur en chef de WES, Richard Brown ${ }^{15}$, insiste sur l'éventail réduit des problèmes qui ont préoccupé les sociologues du travail au cours des années 1950 et 1960, les fameux trois (ou quatre) « $\mathrm{M} »$ :

\footnotetext{
${ }^{14}$ Site Internet de la revue SDT, consulté le 7 janvier 2013.

${ }^{15}$ Richard Brown, né le 28 août 1933, fut président de l'Association britannique de sociologie entre 1983 et 1985. C'est à la suite de son mandat qu'il participa à la création de la revue Work, Employment \& Society, dont il fut rédacteur en chef de 1986 à 1989. Professeur de sociologie et politique sociale à l’Université de Durham entre 1982 et 1993, il publia plusieurs travaux, dont sa thèse, sur le travail féminin dans les manufactures du Nord de l’Angleterre (cf. Beynon 2007).
} 
[...] globalement, jusqu'ici, l'attention (des sociologues du travail) s'est focalisée sur la situation et des expériences des travailleurs manuels masculins manufacturiers et - dans une moindre mesure - de ceux qui les supervisent ou managent (Brown 1987, p. 2).

La création de la revue Work, Employment \& Society est placée sous l'angle de la transformation profonde du monde du travail, notamment associée à l'entrée massive des femmes mariées dans le salariat, ainsi que le développement de nouvelles formes atypiques d'emploi, qui sont explicitement citées comme des évolutions qui mériteraient l'attention renouvelée des chercheur·e·s. Il est frappant de remarquer à quel point l'orientation donnée à TGS dans le contexte français de la fin des années 1990 fait écho aux objectifs assignés à WES une décennie plus tôt en Angleterre... et à quel point $S D T$ passe encore largement sous silence de tels enjeux analytiques et sociaux.

Le contexte sociohistorique de la création de WES a été de toute évidence propice à la reconnaissance de l'importance de l'emploi féminin et des perspectives genre, et la publication de travaux s'inscrivant dans une telle orientation dès les premiers numéros de la revue la place d'emblée sur un sentier d'autant plus 'vertueux' que la consolidation des départements, formations et programmes de recherche en études genre au sein des universités anglo-saxonnes contribuent à alimenter ce courant de recherche tout au long de la période analysée ici (Griffin 2005b). On peut tout à fait imaginer que l'explicitation de telles préoccupations scientifiques ait encouragé les spécialistes des dimensions sexuées du travail à proposer des articles à Work, Employment \& Society, contribuant ainsi à une consolidation de la visibilité des recherches genre en son sein. A contrario, il paraît probable que l'absence de références aux enjeux sexués du travail, combinée à la rareté d'articles portant sur ce thème dans les numéros successifs de Sociologie du travail, ait découragé les spécialistes de ces questions de recherche à proposer leurs articles à la publication dans cette revue.

C’est donc là que les logiques propres au fonctionnement interne des revues scientifiques rencontrent les modalités spécifiques d'institutionnalisation des études genre. Ce n’est pas parce qu'elles ont réussi (avec le soutien de quelques alliés 
masculins de taille) à imposer le genre comme une perspective incontournable au sein même de la sociologie du travail que les chercheures féministes britanniques ont renoncé pour autant à la création parallèle d'autres supports de valorisation de leurs recherches, sous la forme de revues spécialisées 'travail et genre'. L'exemple britannique montre qu'il n'y a aucune incompatibilité entre l'intégration disciplinaire des perspectives de genre et leur professionnalisation / autonomisation. Dans le cas français, c'est la cécité persistante des "gardien·ne·s du temple » disciplinaires à l'égard des dimensions sexuées du travail qui semble avoir motivé la création de revues spécialisées, mais dont l'existence vient, paradoxalement, renforcer, voire justifier l'absence d'intégration des perspectives genre au cœur même des revues généralistes.

Sur le fond, à condition que les recherches sur le genre du travail trouvent un lectorat averti, il importerait peu qu'elles paraissent dans des revues généralistes ou bien explicitement sous la bannière des études genre. Toutefois, malgré la création (et le pénible maintien en circulation) de revues spécialisées (TGS, mais aussi Nouvelles questions féministes, CLIO et les Cahiers du genre), la faible ouverture des revues généralistes aux recherches sur le genre paraît clairement préjudiciable aux chercheur·e's féministes français·es, et les place dans une autre logique institutionnelle que celle qui prévaut en GrandeBretagne. Dans les deux pays, la publication de recherches sur le genre du travail est plus souvent le fait de femmes que d'hommes. Toutefois, dans le contexte britannique, les spécialistes du genre disposent d'infiniment plus de possibilités de valorisation de leurs travaux que leurs homologues françaises. Outre les revues spécialisées, leur domaine de spécialisation ne leur ferme pas pour autant l'accès à l'une des revues généralistes les plus prestigieuses de la sociologie britannique.

Le resserrement de l'horizon des possibles en matière de valorisation des recherches sur le genre du travail dans le contexte français explique sans doute le changement constaté au fil du temps en matière de signatures. Dans le cas britannique, on peut penser que l'indifférenciation des pratiques de cosignature - selon l'appartenance sexuée, mais également selon l’objet ('genre' / 'hors genre') et selon les caractéristiques du 
support de publication (revue généraliste / spécialiste) témoigne de l'aboutissement du processus d'institutionnalisation des études genre selon la modalité de la professionnalisation, voire de la disciplinarisation (Silius 2005). La production scientifique devient collective et cela indépendamment des champs thématiques et des problématiques de recherche. Dans le cas français, les choses paraissent plus ambiguës. En l'absence d'une légitimité forte des recherches sur le genre dans le champ académique dans son ensemble, il se pourrait que les rares occasions de publication sur ce thème dans les revues généralistes soient conditionnées par une rupture visible avec les pratiques de signatures collectives, restées symboliquement associées à la modalité 'activiste' de l'institutionnalisation des études genre, ce qui serait devenu stigmatisant pour les chercheures concernées.

Une dernière hypothèse permettant d'expliquer les évolutions divergentes en matière de signature individuelle ou collective des 'articles genre' concerne les pratiques des comités de rédaction, notamment en matière de confection des numéros successifs des revues. Bien que l'ensemble des revues dont il est question ici affiche une politique d'appels à contribution, l'observation révèle des pratiques passablement divergentes d'un pays à l'autre. Sans doute en raison de l'aire linguistique élargie dans laquelle elles sont susceptibles de puiser, il nous semble que les revues anglo-saxonnes pratiquent plus systématiquement l'appel ouvert à communication, alors que les comités éditoriaux des revues francophones combinent plus fréquemment cette pratique avec la sollicitation directe de contributions auprès de 'spécialistes' connu·e·s et reconnu·e·s des thématiques retenues pour leurs prochaines éditions. Si une telle hypothèse devait se confirmer, elle permettrait d'expliquer la part beaucoup plus importante de contributions signées d'auteur·e·s seul·e·s dans les revues francophones ${ }^{16}$ et également la rareté relative des 'articles genre' en dehors de numéros thématiques dédiés spécifiquement à ce thème, dans le cas français.

Notre exercice comparatif permet donc de conclure que la reconnaissance du genre du travail ne va toujours pas de soi au

\footnotetext{
${ }^{16}$ En général, un·e auteur·e sollicité·e l'est à titre personnel et la contribution attendue de sa part est de nature individuelle.
} 
sein de la sociologie française et que cela est clairement préjudiciable aux chercheur.e's (de plus en plus souvent des femmes, d'ailleurs) qui se lancent dans de telles recherches, alors que le 'stigmate du genre' ne semble pas avoir existé de la même manière dans les milieux académiques équivalents en GrandeBretagne, du moins depuis la fin des années 1980. Il nous reste à explorer ultérieurement les effets de ces configurations variables d'institutionnalisation des études genre sur le contenu même des recherches publiées au fil du temps dans ces deux contextes sociétaux.

Annexe. Évolution de la composition sexuée des Comités de rédaction de quelques revues de sociologie du travail, 1990 - 2012

Tableau A1. Composition sexuée des Comités de rédaction de WES, 1990-2012

\begin{tabular}{|l|c|c|c|}
\hline $\mathbf{1 9 9 0}$ & Hommes & Femmes & \% Femmes \\
\hline Editorial Board & 9 & 8 & $47 \%$ \\
\hline Editorial Advisors & 9 & 4 & $31 \%$ \\
\hline $\mathbf{2 0 1 2}$ & & & \\
\hline Editorial Board & 15 & 15 & $50 \%$ \\
\hline Associate Board & 7 & 16 & $70 \%$ \\
\hline International Advisory Board & 16 & 7 & $30 \%$ \\
\hline
\end{tabular}

Tableau A2. Composition sexuée des Comités de rédaction de SDT, 1990-2012

\begin{tabular}{|l|c|c|c|}
\hline $\mathbf{1 9 9 0}$ & Hommes & Femmes & $\%$ Femmes \\
\hline Comité de rédaction & 6 & 3 & $33 \%$ \\
\hline Comité d'orientation & 6 & 4 & $40 \%$ \\
\hline $\mathbf{2 0 1 2}$ & & & \\
\hline Comité de rédaction & 7 & 9 & $56 \%$ \\
\hline Comité d'orientation & 21 & 0 & $0 \%$ \\
\hline
\end{tabular}

Tableau A3. Composition sexuée des Comités de rédaction de GWO, 1994-2012

\begin{tabular}{|l|r|r|r|}
\hline & Hommes & Femmes & \% Femmes \\
\hline $\mathbf{1 9 9 4}$ & & & \\
\hline Editorial Board & nd & nd & nd \\
\hline Associate Editors & nd & nd & nd \\
\hline $\mathbf{2 0 1 2}$ & & & \\
\hline Editorial Board & 6 & 34 & $85 \%$ \\
\hline Associate Editors & 9 & 69 & $88 \%$ \\
\hline
\end{tabular}

Tableau A4. Composition sexuée des Comités de rédaction de TGS, 1995-2012

\begin{tabular}{|l|l|l|l|}
\hline & Hommes & Femmes & \% Femmes \\
\hline 1999 & & & \\
\hline
\end{tabular}




\begin{tabular}{|l|r|r|r|}
\hline Comité de rédaction & 1 & 6 & $86 \%$ \\
\hline Conseil scientifique & - & - & - \\
\hline $\mathbf{2 0 1 2}$ & & & \\
\hline Comité de rédaction $^{17}$ & 2 & 19 & $90 \%$ \\
\hline Conseil scientifique $^{\mid}$ & 18 & 42 & $70 \%$ \\
\hline
\end{tabular}

${ }^{17}$ Il s’agit du Comité de rédaction dont la composition figure sur le site Internet de la revue. Il existerait également un Comité de rédaction «élargi » à TGS, mais dont la composition n'est pas précisée dans les documents à notre disposition. 


\section{Références}

Abbott Andrew (1999). Department and Discipline. Chicago Sociology at One Hundred. Chicago, University of Chicago Press.

- (2001). Chaos of Disciplines. Chicago, University of Chicago Press.

Andriocci Muriel, Le Feuvre Nicky (2006). « Les enjeux sociaux de l'institutionnalisation des 'études féministes' à l'Université ». In Ollagnier Edmée, Solar Claudie (eds).

Badinter Élisabeth (2003). Fausse route : réflexions sur 30 années de féminisme. Paris, Odile Jacob.

Bataille Pierre (2013). «Quel goût pour les sciences ? Une question d'interprétation et de méthode ». LIVES Working Papers, ${ }^{\circ} 20.4$ : www.lives-nccr.ch/fr/page/lives-working-papers-list-papers-n78

Bereni Laure (2011). « Genre : état des lieux ». Entretien avec Mathieu Trachman. La Vie des idées, 5 octobre : www.laviedesidees.fr/Genre-etat-des-lieux.html

- (2012). «Une nouvelle génération de chercheuses sur le genre. Réflexions à partir d'une expérience située ». Contretemps, $\mathrm{n}^{\circ} 20$, juin : www.contretemps.eu

Beynon Huw (2007). "Richard Brown. A Sociologist whose Research Looked at the Wartime Experience of Women Workers". The Guardian, 14 juin.

Borzeix Anni, Rot Gwenaële (2010). Genèse d'une discipline, naissance d'une revue. 'Sociologie du travail'. Nanterre, Presses universitaires de Paris Ouest.

Brown Richard (1987). "Work, Employment \& Society: An Editorial Introduction”. Work, Employment \& Society, vol. 1, $\mathrm{n}^{\circ} 1$.

Chenu Alain (2002). « Une institution sans intention. La sociologie en France depuis l'après-guerre ». Actes de la recherche en sciences sociales, vol. $1, \mathrm{n}^{\circ} 141-142$.

CLIO. Histoire, femmes et sociétés (2002). «L'Histoire des femmes en revues France-Europe. Regards complémentaires », n 16.

Commission européenne (2009). She Figures 2009. Statistics and Indicators on Gender Equality in Science. Bruxelles, DG Recherche et innovation.

David Paul A. (1985). "Clio and the Economics of QWERTY". The American Economic Review, vol. 75, n 2.

Ezekiel Judith (1994) «Pénurie de ressources ou de reconnaissance? Les études féministes en France ». Bulletin de l'ANEF, n 16. 
Fassa Farinaz, Kradolfer Sabine (eds) (2010). Le plafond de fer de l'Université : femmes et carrières. Genève, Seismo.

Fougeyrollas-Schwebel Dominique, Planté Christine, Riot-Sarcey Michèle, Zaidman Claude (eds) (2003). Le genre comme catégorie d'analyse. Sociologie, histoire, littérature. Paris, L'Harmattan.

Galtung Jon (1982). “On the Meaning of 'Nation' as a Variable”. In Niessen Manfred, Peschar Jules (eds). International Comparative Research: Problems of Theory, Methodology and Organization in Eastern and Western Europe. Oxford, Pergamon Press.

Griffin Gabriele (ed) (2004). Employment, Equal Opportunities, and Women's Studies. Women's Experiences in Seven European Countries. Berlin, Helmer Verlag.

- (2005a). Doing Women's Studies: Employment Opportunities, Personal Impacts and Social Consequences. London, Zed Books.

- (2005b). "The institutionalization of Women's Studies in Europe”. In Griffin (ed).

Guilbert Madeleine (1966). Les fonctions des femmes dans l'industrie. La Haye, Mouton.

Lallement Michel (2003). «Quelques remarques à propos de la place du genre dans la sociologie du travail en France ». In Laufer Jacqueline, Marry Catherine, Maruani Margaret (eds). Le travail du genre. Paris, La Découverte.

Le Feuvre Nicky (2010). «Les carrières universitaires à l'épreuve du genre : éléments de conclusion ». In Fassa Farinaz, Kradolfer Sabine (eds).

Le Feuvre Nicky, Andriocci Muriel (2004). “The Impact of Women's Studies on Women's Employment Opportunities and Experiences in France”. In Griffin Gabriele (ed).

- (2005). "Employment Opportunities for Women in Europe". In Griffin Gabriele (ed) (2005a).

Maruani Margaret (2001). «L'emploi féminin dans la sociologie du travail : une longue marche à petits pas ». In Laufer Jacqueline, Marry Catherine, Maruani Margaret (eds). Masculin-féminin : questions pour les sciences de l'Homme. Paris, Presses universitaires de France.

Musselin Christine (2005). Le marché des universitaires. France, Allemagne, États-Unis. Paris, Presses de Sciences Po.

Myrdal Alva, Klein Viola (1956). Women's Two Roles: Home and Work. London, Routledge \& Kegan Paul.

Ollagnier Edmée, Solar Claudie (eds) (2006). Parcours de femmes à l'Université : perspectives internationales. Paris, L'Harmattan «Genre et éducation ». 
Ozouf Mona (1995). Les mots des femmes : essai sur la singularité française. Paris, Fayard.

Pierson Paul (1994). Dismantling the Welfare State? Reagan, Thatcher, and the Politics of Retrenchment. Cambridge, Cambridge University Press.

Puig de la Bellacasa María (2002). "Gender Relations in the Flexible University”. In Passerini Luisa, Lyon Dawn, Borghi Liana (eds). Gender Studies in Europe. Florence, European University Institute, Robert Schumann Centre.

Silius Harriet (2002). “Women’s Employment, Equal Opportunities and Women's Studies in Nine European Countries: A Comparative Summary”. In Griffin Gabriele (ed). Women's Employment, Women's Studies and Equal Opportunities, 1945-2001. Reports from Nine European Countries. Hull, University of Hull Press.

- (2005). "The Professionalization of Women’s Studies in Europe: Expectations and Experiences”. In Griffin Gabriele (ed) (2005a). 